\title{
Actividad antioxidante y ácidos grasos de aceite de semillas de siete frutas nativas de la región Amazonas, Perú
}

\author{
Rosita Cruz ${ }^{1}$, Segundo G. Chavez ${ }^{2^{\star}}$ y Armstrong B. Fernández-Jeri² \\ (1) Facultad de Ingeniería y Ciencias Agrarias, Escuela Profesional de Ingeniería Agroindustrial, Universidad Nacional \\ Toribio Rodríguez de Mendoza de Amazonas, Calle Higos Urco 350, Chachapoyas-Perú. \\ (Correo-e: rosita.cruz@untrm.edu.pe) \\ (2) Instituto de Investigación, Innovación y Desarrollo para el Sector Agrario y Agroindustrial de la región Amazonas, \\ Universidad Nacional Toribio Rodríguez de Mendoza de Amazonas, Calle Higos Urco 350, Chachapoyas-Perú. \\ (Correo-e: segundo.quintana@untrm.edu.pe, armstrong.fernandez@untrm.edu.pe)
}

*Autor a quien debe ser dirigida la correspondencia.

Recibido Nov. 28, 2020; Aceptado Ene. 25, 2021; Versión final Mar. 4, 2021, Publicado Jun. 2021

\begin{abstract}
Resumen
El principal objetivo de esta investigación fue determinar la actividad antioxidante y el potencial agroindustrial de aceites extraídos de semillas de la región Amazonas (Perú) de siete frutos distintos: Vasconcellea pubescens, Vasconcellea weberbaueri (Harms) V.M, Solanum betaceum, Passiflora edulis, Physalis peruviana, Passiflora tripartita y Spondias purpurea L. Los aceites se extrajeron usando la técnica Soxhlet. Se encontró que los aceites de las semillas de $V$. pubescens, $V$. weberbaueri harms, $S$. betaceum, $P$. edulis, $P$. peruviana, y $P$. tripartita tienen potencial para usarse en el desarrollo de alimentos $(\mathrm{IS}=62 \mathrm{a} 84 ; \mathrm{IA}=0.18$ a $0.64 \%)$. Se observó heterogeneidad en la estabilidad oxidativa. El aceite de $V$. weberbaueri fue el más estable $(I P=16.13)$ y el de $V$. pubescens $(I P=126.34)$ fue el más inestable. Se concluye que los aceites examinados tienen un elevado potencial para ser empleados en alimentación humana, en desarrollo de alimentos, en desarrollo de biocombustibles y en otras aplicaciones no alimentarias.
\end{abstract}

Palabras clave: aceites; actividad antioxidante; peróxidos; semillas; potencial agroindustrial

\section{Antioxidant activity and fatty acids of seed oil of seven native fruits of the Amazon region, Peru}

\begin{abstract}
The primary objective of this research study was to determine the antioxidant activity and the agro-industrial potential of oils extracted from seven different Amazon (Peru) fruit seeds: Vasconcellea pubescens, Vasconcellea weberbaueri (Harms) V.M., Solanum betaceum, Passiflora edulis, Physalis peruviana, Passiflora tripartita, and Spondias purpurea L. The Soxhlet technique was used for extraction of seed oils. The results showed that $V$. pubescens, $V$. weberbaueri harms VM, S. betaceum, $P$. edulis, $P$. peruviana, and $P$. tripartita seed oils have potential to be used in food development (IS $=62$ to 84 ; $\mathrm{Al}=0.18$ to $0.64 \%$ ). Heterogeneity was also observed in oxidative stability. The $V$. weberbaueri seed oil was the most stable $(I P=16.13)$ while the $V$. pubescens seed oil was the most unstable $(I P=126.34)$. It is concluded that the seed oils examined have great potential for human consumption, for developing foods and biofuels, and for developing other nonfood related applications.
\end{abstract}

Keywords: oils; antioxidant activity; peroxides; seeds: agro-industrial power 


\section{INTRODUCCIÓN}

En la actualidad, los consumidores son cada vez más exigentes al momento de adquirir productos transformados, haciendo que la industria alimentaria tenga la necesidad de sustituir o en todo caso disminuir los componentes de origen sintético, que a largo plazo pueden causar daños en la salud. Lo cual está impulsando la búsqueda de nuevas fuentes naturales, así se han estudiado muchos extractos obtenido de plantas con capacidad de retardar la oxidación en el organismo humano (Coultate, 2002), los que contienen moléculas capaces de prevenir el deterioro químico por oxidación de las células. Estas moléculas pueden ser empleados, también en la industria alimentaria como aditivos por su capacidad de retardar el deterioro oxidativo de los alimentos. En los aceites dilatan el proceso de rancidez, evitan la reacción de las enzimas oxidativas, inhiben la producción de malos olores y además se han demostrado que actúan también como agentes antimicrobianos (Nazzaro et al., 2019; Seukep, 2020). También, las propiedades nutricionales y terapéuticas han sido ampliamente demostradas y, el sólo hecho de identificar su presencia o su actividad confiere al alimento un mayor valor en el mercado (Cuerda et al., 2011).

Uno de los grupos de compuestos con alto valor nutricional, son los ácidos grasos que según su fuente y naturaleza pueden ser muy bien valorados (Holub, 2002). Además, son fundamentales en la dieta (omega 3 y 6) (Saini y Keum, 2018), cuya deficiencia pueden causar serias enfermedades (Jones, 2002). Se han reportado estudios sobre la identificación y valoración de subproductos como fuente de moléculas antioxidantes (Murga et al., 2002), los que han permitido que muchos subproductos se incluyan en los procesos industriales, así por ejemplo se ha logrado utilizar la "torta de orujo" para extraer compuestos que mejoran la estabilidad de aceites comestibles (Abd et al., 2010) y restos de los procesos de la industria del vino son usados como materia para obtener antioxidantes que posteriormente son utilizados para la conservación durante los procesos que implican congelar y cocer la carne (Selani et al., 2011).

En consecuencia, se evidencia un creciente interés por la búsqueda de fuentes no convencionales de aceites con alto valor nutricional; así por ejemplo, se ha determinado que los aceites extraídos de semillas de naranja (Citrus sinensis) contiene compuestos bioactivos con elevado potencial antioxidante (Neuza et al., 2016; Sümeye y Bastürk, 2019). También, su estudio permite identificar las potenciales aplicaciones en la industria, por ejemplo, dada su composición, algunas semillas pueden ser fuente de aceites con uso alimentario, farmacéutico, cosmético, como biocombustible e incluso ser empleados en la fabricación de piensos para la alimentación animal (Lafont et al., 2019; Pantoja-Chamorro et al., 2017).

Una de las fuentes ricas en aceites de gran valor (omegas), son las semillas de las frutas, las que en su mayoría no se aprovechan (en la industria) o si son consumidas, no se conoce su potencial nutritivo. Así tenemos, dentro de muchas otras, a las semillas de los frutos de maushan (Vasconcellea weberbaueri Harms V.M. Badillo), maracuyá (Passiflora edulis), pur pur (Passiflora tripartita), tomate de árbol (Solanum betaceum), aguaymanto (Physalis peruviana), papayita de monte (Vasconcellea pubescens) y ciruela (Spondias purpurea L.). Estas frutas son muy consumidas en las zonas interandinas del Perú y recientemente están siendo empleadas en la industria agroalimentaria, en dónde las semillas y cáscaras son generalmente desechadas o subutilizadas. Aún no se ha reportado estudios sobre la valoración de las semillas de estas frutas como posibles fuentes de aceites con potencial antioxidante.

La calidad de los aceites de semillas está dada por sus propiedades que posee; así tenemos a la actividad antioxidante, la estabilidad a la oxidación (formación de peróxidos), el índice de refracción como indicador de pureza y la capacidad de saponificación como indicador de la cantidad y calidad de ácidos grasos (Coultate, 2002; Jones, 2002). Con excepción de las semillas de $P$. peruviana, los trabajos previos abordan la caracterización total o parcial del fruto (de interés comercial inmediato), por lo que su estudio amplía el conocimiento sobre la naturaleza de las semillas como fuente de aceites, generalmente consideradas como subproductos y desechadas por la industria alimenticia (Chau y Huang, 2004; Contreras-Calderon et al., 2011; Ramadan y Morsel, 2008). Por lo tanto, el objetivo de la investigación fue determinar la actividad antioxidante y el potencial agroindustrial de aceites extraídos de semillas de siete frutos distintos (Vasconcellea pubescens, Vasconcellea weberbaueri harms V.M, Solanum betaceum, Passiflora edulis, Physalis peruviana, Passiflora tripartita y Spondias purpurea L.) de la región Amazonas.

\section{METODOLOGÍA}

Los frutos fueron recolectados de diferentes lugares de la región Amazonas, ubicada al nororiente del Perú, cuyas coordenadas se detalla en la Tabla 1. En Laboratorio de Tecnología de los Alimentos de la Universidad Nacional Toribio Rodríguez de Mendoza de Amazonas, las semillas fueron identificadas, separadas, lavadas con agua destilada y secadas en estufa a $50^{\circ} \mathrm{C}$ durante $12 \mathrm{~h}$ hasta obtener humedades entre 12 y $14 \%$. Luego fueron envasadas al vacío en bolsas de polietileno de alta densidad para protegerlas de la humedad y algún otro factor de deterioro, hasta su posterior procesamiento. 
Tabla 1: Lugares georeferenciados de colecta de los frutos fuente de semillas oleaginosas.

\begin{tabular}{|c|c|c|c|}
\hline Lugar & $\begin{array}{l}\text { Altitud } \\
\text { (msnm) }\end{array}$ & Coordenadas & Especie colectada \\
\hline \multirow{2}{*}{ Choctamal } & 2748 & S 06²3.616', O $077^{\circ} 59.189^{\prime}$ & V. weberbaueri \\
\hline & 2638 & S 06²3.394', O 077'59.294' & V. pubescens \\
\hline \multirow{2}{*}{ Levanto } & 2476 & S 06¹4.509', O 077’51.550' & P. tripartita \\
\hline & 2659 & S 06¹8.443', O 077’54.019' & S. betaceum \\
\hline María & 2772 & S 06²5.882', O 077’57.437' & P. peruviana \\
\hline Bagua Grande & 648 & S 0554.008', O 078'07.895' & S. purpurea \\
\hline Jamalca & 794 & S 06¹3.995', O 077'51.277' & P. edulis \\
\hline
\end{tabular}

\section{Proceso de extracción de los aceites}

Se empleó la técnica de extracción con solvente (Stupp et al., 2008). Para lo cual, fue necesario triturar las semillas en un mortero de porcelana. Para triturar las semillas de ciruela, debido a su dureza, se utilizó un molino mecánico manual para granos; obteniendo $100 \mathrm{~g}$ de muestra de cada tipo de semilla. Posteriormente se procedió a extraer las grasas y aceites en un equipo Soxhlet empleando éter de petróleo como solvente. Se ejecutaron 10 sifonadas por cada muestra a $200^{\circ} \mathrm{C}$ durante 4 horas. Se separó el solvente del aceite por evaporación en estufa a $50^{\circ} \mathrm{C}$.

\section{Determinación de la actividad antioxidante}

La actividad antioxidante de los aceites extraídos se determinó siguiendo el procedimiento descrito por Chen y Yen (2007) y Muñoz et al. (2010). Para ello se preparó $100 \mathrm{~mL}$ de solución de DPPH (2,2-difenil-1-picril hidrazilo) en metanol $50 \mathrm{mg} / \mathrm{L}$. Luego se preparó una solución de metanol y los extractos en una concentración de $20 \mathrm{mg} / \mathrm{mL}$, que fue la solución A. Para el blanco de muestra se preparó $0.75 \mathrm{~mL}$ de muestra de la solución A y $1.5 \mathrm{~mL}$ de metanol. Posteriormente se preparó el patrón de referencia con $1.5 \mathrm{~mL}$ de DPPH y $0.75 \mathrm{~mL}$ de agua ultra pura. En seguida se preparó la muestra con $0.75 \mathrm{~mL}$ de solución A y de DPPH $1.5 \mathrm{~mL}$, se dejó en reposo por un tiempo de 5 minutos. Se midió las absorbancias en espectrofotómetro UV-vis a una longitud de $515 \mathrm{~nm}$. Con los valores obtenidos de la absorbancia se calculó el porcentaje de captación de radicales libres (Mesa-Vanegas et al., 2015). Para ello se utilizó la siguiente formula (ec. 1). Donde A1: Absorbancia del patrón de referencia; A2: Absorbancia de la muestra; A3: Absorbancia del blanco de muestra.

Actividad antioxidante $=\left[1-\frac{\mathrm{A} 2-\mathrm{A} 3}{\mathrm{~A} 1}\right] * 100$

\section{Determinación de índice de saponificación}

En un matraz Erlenmeyer de $250 \mathrm{~mL}$, se colocó $5 \mathrm{~g}$ de aceite; luego, se añadió $50 \mathrm{~mL}$ de solución alcohólica de hidróxido de potasio $(\mathrm{KOH})$ al $10.5 \mathrm{~N}$ y se sometió a reflujo en un sistema Soxhlet, durante $30 \mathrm{~min}$ (procesos de saponificación) (Chaterjea y Shinde, 2012). Luego se dejó enfriar, para proceder a titular el KOH residual con cloruro de hidrógeno $(\mathrm{HCl}) 0.5 \mathrm{~N}$. Se hace una determinación en blanco. Finalmente se calculó usando la siguiente ecuación (2). Donde $\mathrm{V}_{1}$ : $\mathrm{HCl}$ gastado en la valoración del ensayo en blanco $(\mathrm{mL}) ; \mathrm{V}_{2}: \mathrm{HCl}$ gastado en la valoración de la muestra $(\mathrm{mL}) ; \mathrm{N}$ : normalidad de la solución $\mathrm{HCl}(0.5 \mathrm{~N})$; G: peso de la muestra en gramos $(\mathrm{g})$.

$$
\mathrm{IS}=\frac{\left(\mathrm{V}_{1}-\mathrm{V}_{2}\right) \times N \times 56,1 \mathrm{~g} / \mathrm{eq}}{\mathrm{G}}
$$

\section{Determinación de índice de refracción}

Se colocó una gota de agua destilada para calibrar el refractómetro tipo Abbe (2WAJ), posteriormente se colocó una gota de muestra de aceite en la lámina del refractómetro y se cerró al instante, para luego de un minuto hacer la lectura respectiva.

\section{Determinación de índice de acidez}

Se pesó $4 \mathrm{~g}$ de muestra debidamente homogeneizada en un matraz Erlenmeyer de $250 \mathrm{~mL}$. Luego se añadió $50 \mathrm{~mL}$ de etanol, para posteriormente agregar unas gotas de indicador de fenolftaleína. Se procedió a titular con $\mathrm{NaOH} 0.1 \mathrm{~N}$ hasta lograr que la solución tome un ligero color rosa. El gasto del hidróxido de sodio se 
anotó y se calculó usando la siguiente ecuación (3). Donde G: Gasto de hidróxido de sodio, N: normalidad de tiosulfato, W: peso de la muestra.

$$
\mathrm{IA}=\frac{\mathrm{G} \times \mathrm{N} \times 56,1}{\mathrm{~W}}
$$

\section{Determinación de índice de peróxido}

Las muestras de aceite fueron pesadas en un matraz Erlenmeyer de $250 \mathrm{~mL}(0.062 \mathrm{~g})$. Se le añadió $30 \mathrm{~mL}$ de solución $\mathrm{HOAC}_{-} \mathrm{CHCl}_{3}$ y se agitó hasta lograr disolver por completo. Luego se añadió $15 \mathrm{~mL}$ de ácido acético y $0.5 \mathrm{~mL}$ de $\mathrm{KI}$, se agitó y dejó por 2 min en ambiente oscuro; posteriormente, se añadió $30 \mathrm{~mL}$ de agua destilada. Se añadió $0.5 \mathrm{~mL}$ de solución de almidón al $1 \%$ y en seguida se valoró el yodo liberado con tiosulfato $0.1 \mathrm{~N}$ (Deng et al., 2018). Se corrió un blanco conjuntamente con la muestra. Para los cálculos se aplicó la siguiente ecuación (4). Donde IP: índice de peróxido, V: $\mathrm{mL}$ de solución valorada de tiosulfato de sodio, N: normalidad de la solución de tiosulfato y P: peso de la muestra de aceite.

$$
\mathrm{IP}=\frac{\mathrm{VxN} \times 1000}{\mathrm{P}}
$$

\section{Análisis de datos}

Para el procesamiento de los datos obtenidos a nivel descriptivo, se utilizó medias, tablas y gráficos propios de la estadística descriptiva de análisis univariado. Para evaluar la diferencia entre la actividad antioxidante de las muestras de siete especies se empleó la prueba paramétrica de análisis de varianza (ANOVA) y comparaciones múltiples de Duncan, con un nivel de significancia de 0.05 , utilizando el paquete estadístico SPSS V.26.

\section{RESULTADOS}

Los aceites obtenidos de las distintas semillas tuvieron características distintas. Los aceites de S. betaceum y $P$. tripartita tuvieron mayor actividad antioxidante y baja acidez, sin embargo, también se observa elevados valores de índice de peróxido. El aceite menos propenso a la oxidación fue el de semillas de $V$. weberbaueri y el que mayor oxidación tuvo fue el de semillas de $V$. pubescens (Tabla 1).

Tabla 1: Valores promedios de índice de refracción, actividad antioxidante, índice de saponificación, índice de acidez e índice de peróxido de siete semillas de frutas.

\begin{tabular}{|l|c|c|c|c|c|}
\hline \multicolumn{1}{|c|}{ Especie } & $\begin{array}{c}\text { Índice de } \\
\text { refracción }\end{array}$ & $\begin{array}{c}\text { Actividad } \\
\text { antioxidante } \\
\text { (\% captura DPPH) }\end{array}$ & $\begin{array}{c}\text { Índice de } \\
\text { saponificación } \\
(\mathrm{mg} \mathrm{KOH/g} \mathrm{de} \mathrm{grasa)})\end{array}$ & $\begin{array}{c}\text { Índice de } \\
\text { acidez } \\
\text { (\%) }\end{array}$ & $\begin{array}{c}\text { Índice de peróxido } \\
\text { (meq O2/kg de } \\
\text { grasa) }\end{array}$ \\
\hline P. edulis & 1.46 & 39.08 & 84.55 & 0.16 & 47.85 \\
\hline P. peruviana & 1.70 & 19.70 & 72.38 & 0.21 & 64.52 \\
\hline V. weberbaueri Harms & 1.71 & 21.02 & 84.29 & 0.68 & 16.13 \\
\hline P. tripartita & 1.65 & 46.63 & 62.02 & 0.37 & 73.66 \\
\hline S. petaceum & 1.65 & 62.23 & 69.29 & 0.28 & 95.16 \\
\hline V. pubescens & 1.68 & 35.14 & 66.87 & 0.23 & 126.34 \\
\hline S. purpurea & 1.67 & 15.93 & 15.88 & 0.16 & 80.65 \\
\hline
\end{tabular}

\section{Capacidad antioxidante}

Se encontró diferencias significativas $(\mathrm{p}=0.05)$ en la actividad antioxidante de los aceites extraídos de siete semillas de frutos distintos (Figura 1). El aceite extraído de semillas de $S$. betaceum tuvo la mayor actividad antioxidante (prueba Duncan, $p=0,05$ ), luego hay un segundo grupo que podría catalogarse como de actividad antioxidante media (35-46\%) conformado por los aceites extraídos de $P$. tripartita, $P$. edulis y $V$. pubescens. El tercer grupo y con menor actividad antioxidante fueron los aceites de semillas de $V$. weberbaueri Harms, $P$. peruviana y $S$. purpurea (19-21\%). Consecuentemente, los aceites extraídos de las semillas de las siete especies frutales tienen una muy variada actividad antioxidante (15 hasta $62 \%$ ) y la semilla cuyo aceite tuvo mayor valor, es una semilla generalmente consumida con la fruta en fresco de $S$. betaceum. Por otro lado, observamos que el valor antioxidante más bajo, fue del aceite de la semilla de $S$. purpurea que de ningún modo puede ser considerada comestible. 


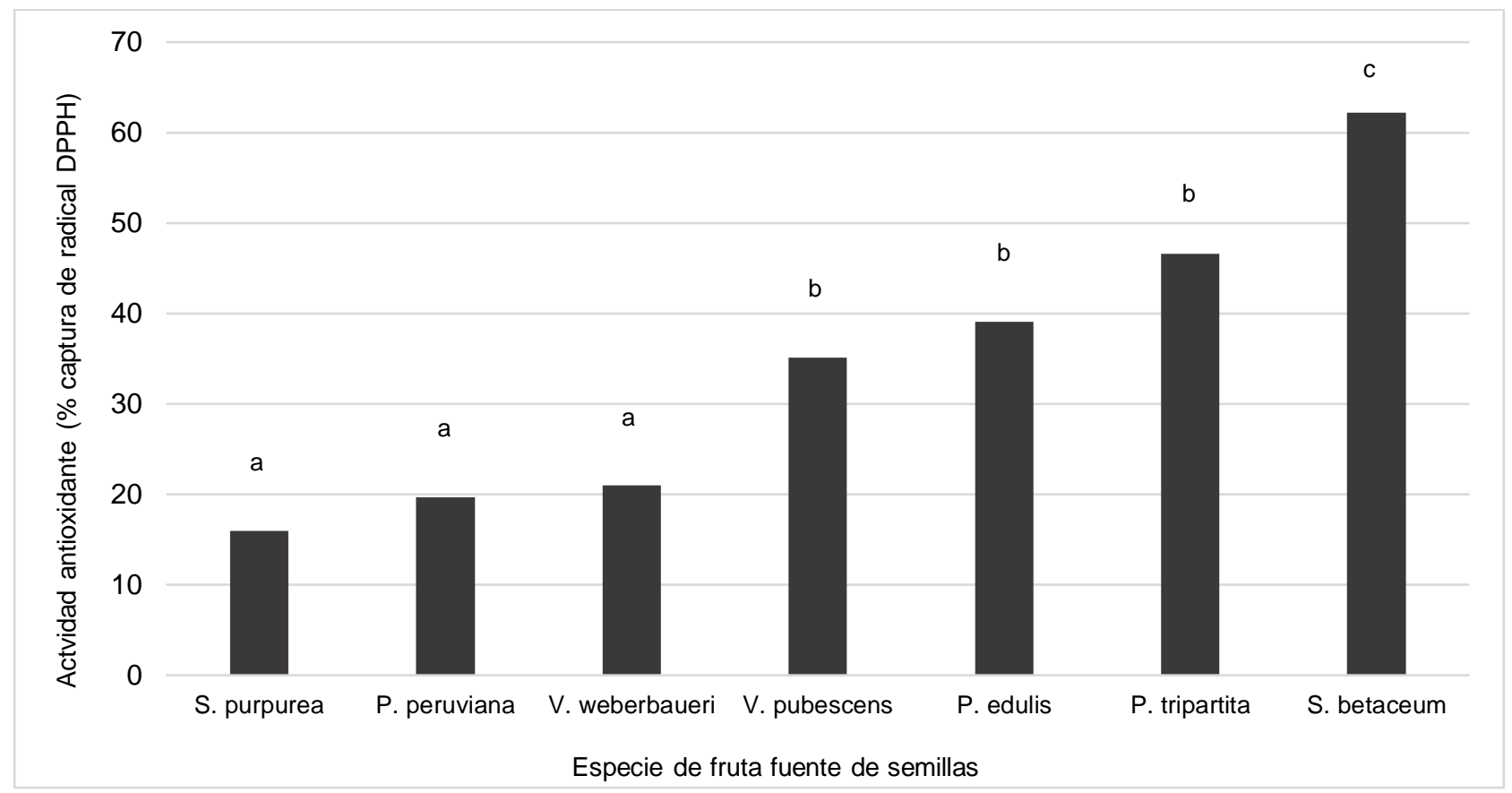

Fig. 1: Capacidad antioxidante de aceites extraídos de siete semillas distintas. Letras indican grupos diferentes.

\section{Índice de saponificación}

Se encontró diferencias significativas $(\mathrm{p}=0.05)$ en los índices de saponificación de los aceites extraídos de siete semillas de frutos distintos (Figura 2). Todos los aceites tuvieron un elevado índice de saponificación (>60) con excepción de $S$. purpurea (15). Tal como se observa en la Tabla 1, hay seis grupos bien diferenciados $(p=0,05)$ y los dos aceites con mayor índice de saponificación tiene valores estadísticamente iguales $(P$. edulis y $V$. weberbaueri) con más de 84 . De manera similar a la actividad antioxidante, el aceite de $S$. pupurea tuvo menor índice de saponificación y los aceites de semillas comestibles obtuvieron valores hasta cuatro veces superiores a ésta (Tabla 1y Figura 2).

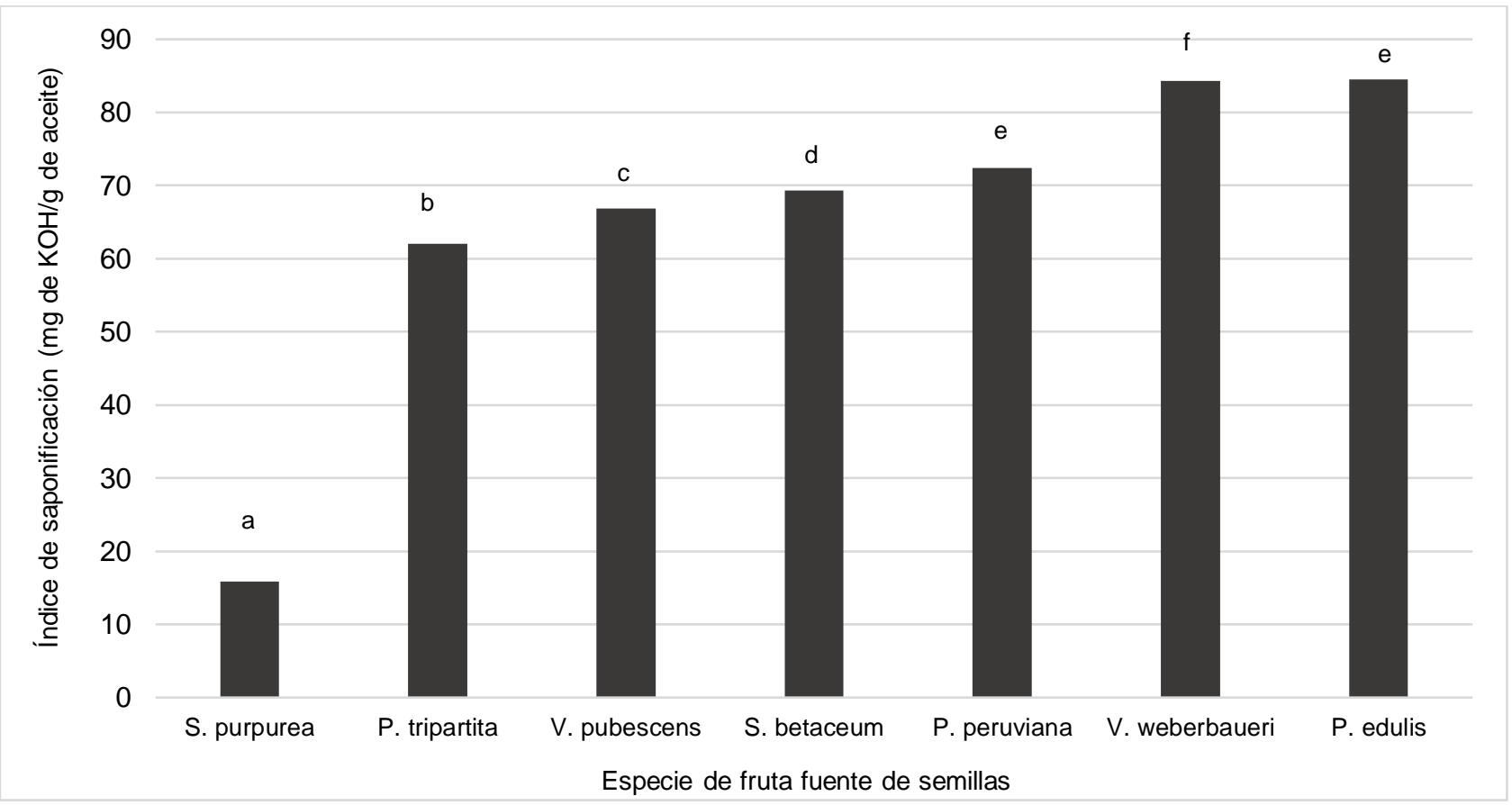

Fig. 2: Índice de saponificación de aceites extraídos de siete semillas distintas. Letras indican grupos diferentes 


\section{Índice de refracción}

No se encontró diferencias significativas $(\mathrm{p}=0.05)$ en los índices de refracción de los aceites extraídos de las siete semillas y los valores oscilaron entre 1.46 y 1.71 (Tabla 1 ).

Índice de acidez

Se encontró diferencias significativas $(p=0.05)$ en los índices de acidez de los aceites extraídos de siete semillas de frutos distintos (Figura 3 y Tabla 1). Sin embargo, todos los aceites tuvieron niveles de acidez muy bajos (indicadores de baja oxidación). Teniendo en cuenta que mientras más bajo índice de acidez mejor será el aceite. Las semillas de $S$. purpurea tuvieron aceites más estables e iguales a $P$. edulis (grupo 1), seguidos por P. peruviana y V. pubescens (grupo 2), luego $S$. betaceum (grupo 3), P. tripartita (grupo 4) y finalmente V. weberbaweri (grupo 5).

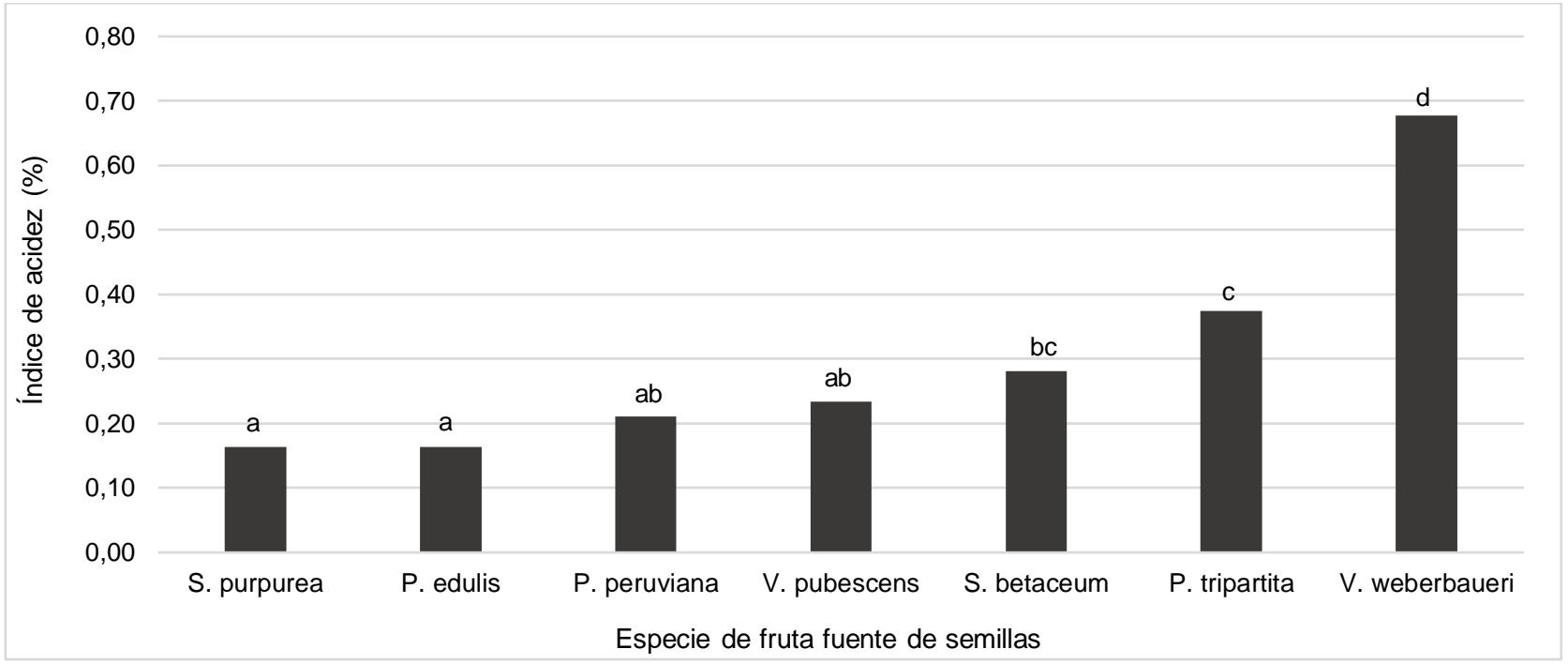

Fig. 3: Índice de acidez de aceites extraídos de siete semillas distintas. Letras diferentes indican grupos diferentes.

\section{Índice de peróxido}

Se encontró diferencias significativas $(p=0,05)$ en los índices de peróxido de los aceites extraídos de semillas de siete frutos distintos (Figura 4 y Tabla 1). Los valores calculados, permite conocer la estabilidad de cada uno de los aceites estudiados. El aceite más estable fue el extraído de semillas de $V$. weberbaueri (16.13 meq $\mathrm{O}_{2} / \mathrm{kg}$ ) y el más inestable (o más oxidado), fue el aceite extraído de las semillas de $V$. pubescens (126.34 meq $\mathrm{O}_{2} / \mathrm{kg}$ ). Se encontró siete grupos bien distribuidos mediante la prueba estadística de comparaciones múltiples de Duncan, tal como se puede observar en la Figura 4.

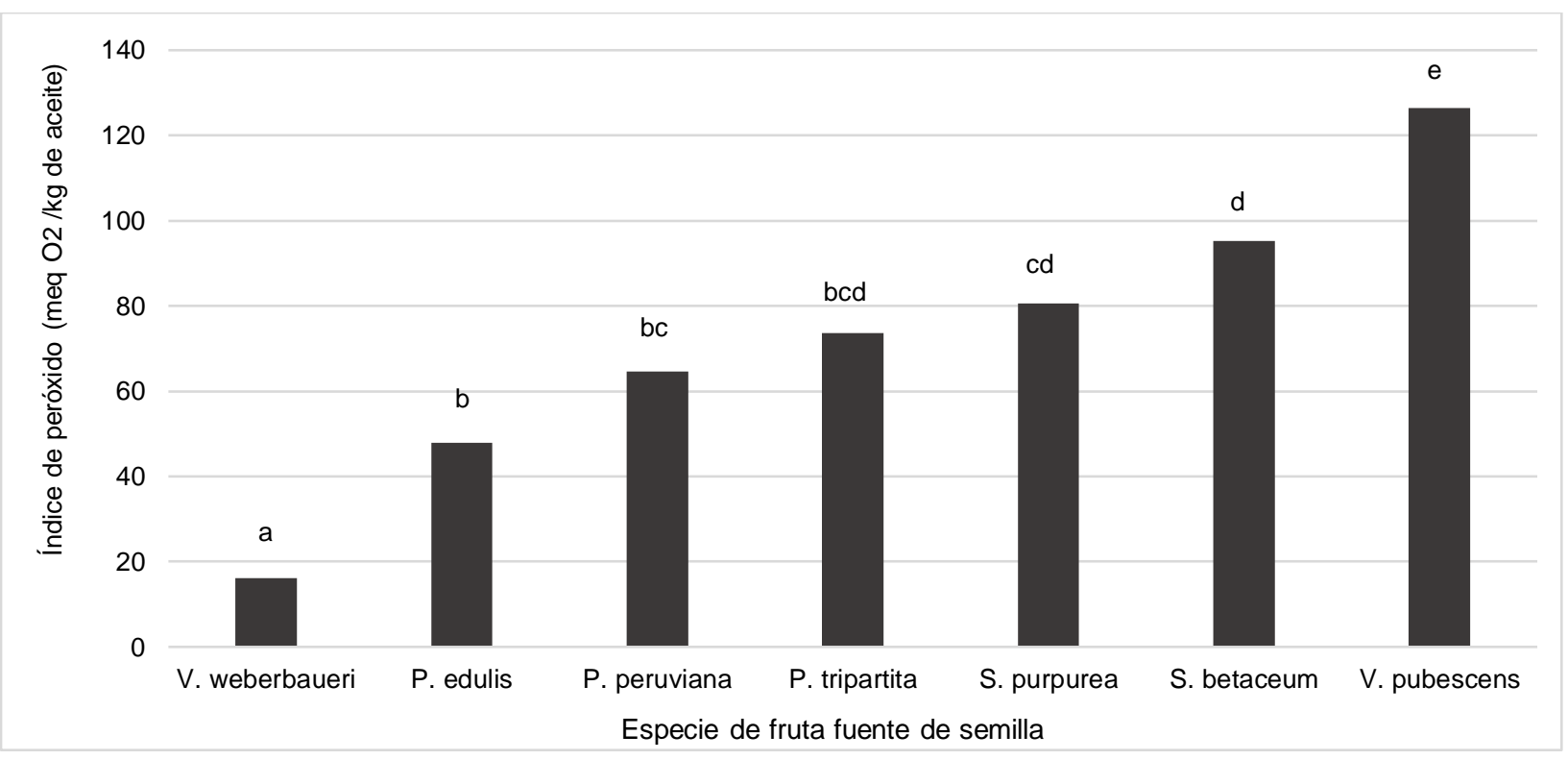

Fig. 4: Índice de peróxido de aceites extraídos de siete semillas distintas. Letras diferentes indican grupos diferentes. 


\section{DISCUSIÓN}

El aceite de semilla de $S$. betaceum, tuvo la mayor actividad antioxidante en el ensayo in vitro, teniendo en cuenta que ésta fruta es generalmente consumida con las semillas incluidas, esta información aporta a los conocimientos que se tiene sobre las bondades de la fruta (pulpa y zumo), cuya capacidad antioxidante ha sido muy estudiada. Debido a que los componentes con mayor actividad antioxidante presentes en los aceites vegetales, son los tocoferoles y otros fenoles (Al Juhaimi y Özcan, 2018; Ghafoor et al., 2019); podría suponerse que el aceite de semilla de $S$. betaceum es rico en estos compuestos.

El aceite de semillas de S. purpurea, tuvo baja actividad antioxidante y bajo índice de saponificación, indicador claro de su bajo potencial para la industria alimentaria; no obstante, podría contener otros compuestos no grasos con relevancia alimenticia (Franco-Mora et al., 2015). También puede ser evaluado como fuente para la obtención de biocombustibles (Benavides et al., 2007). Todos los aceites presentaron índice de acidez menores al 1\%, valor límite para los aceites extra vírgenes según Normas del Codex Alimentarius, claro indicador de que los aceites de las siete semillas estudiadas son de muy buena calidad y que si se emplearía técnicas de extracción a menores temperaturas podría obtenerse mejores resultados aún. Sin embargo, mayores estudios como análisis proximal, determinación del perfil de ácidos grasos y su estabilidad al almacenamiento, para determinar con mayor precisión su aplicación industrial (Lafont et al., 2019) en la cosmética, farmacéutica o alimentaria (Pantoja-Chamorro et al., 2017) e incluso en algunos caso podría no ser necesaria la extracción de los aceites, sino ser considerados como insumos potenciales para la elaboración de piensos compuestos (Lafont et al., 2011).

Los valores elevados de índice de peróxido en todos los aceites evaluados ( $>16$ meq $\mathrm{O}_{2} / \mathrm{kg}$ ), podría deberse a la técnica de extracción, debido a que Soxhlet requiere de calentamiento para recircular el solvente, por lo tanto la extracción no se realiza en frío, como sí se hace en los aceites extra virgen de otras fuentes (Al Juhaimi y Özcan, 2018). Sólo el aceite extraído de las semillas de V. weberbaweri, tuvo un índice de peróxido cercano al límite determinado para aceite de oliva estipulado por la Comisión Delegada de Regulación de la Unión Europea (2019) en 20 meq O2/kg y para aceite de sachainchi producido en Perú hasta 17.47 meq O2/kg (Ramos-Escudero et al., 2019).

\section{CONCLUSIONES}

Debido a su actividad antioxidante, los aceites extraídos de las semillas de $V$. pubescens, $V$. weberbaweri, $S$. betaceum, $P$. edulis, $P$. peruviana, $P$. tripartita, tienen un elevado potencial para ser empleados en alimentación humana y desarrollo de alimentos, y; el aceite extraído de las semillas de $S$. purpurea, con mayores estudios podría ser empleado en el desarrollo de biocombustibles u otras aplicaciones no alimentarias. Los siete aceites evaluados tuvieron índices de acidez, refracción y saponificación dentro del límite estipulado por la Norma Técnica Peruana para aceites vírgenes. Sólo el aceite extraído de semillas de $V$. weberbaweri, obtuvo un índice de peróxido menor a $20 \mathrm{meq} \mathrm{O}_{2} / \mathrm{kg}$ establecido por la Unión Europea, lo cual le confiere una elevada estabilidad oxidativa.

\section{REFERENCIAS}

Abd, G., Ammar, M.S., y Hegazy, A.E., Use of olive waste cake extract as a natural antioxidant for improving the stability of heasted sunflower oil, World Applied Sciences Journal, 11(1) 106-113 (2010)

Al Juhaimi, F., y Özcan, M.M., Effect of cold press and soxhlet extraction systems on fatty acid, tocopherol contents, and phenolic compounds of various grape seed oils, https://doi.org/10.1111/jfpp.13417, Journal of Food Processing and Preservation, 42(1), e13417 (2018)

Benavides, A., Benjumea, P., y Pashova, V., El biodiesel de aceite de higuerilla como combustible alternativo para motores diesel, Ideas y Valores, 74(153), 141-150 (2007)

Chaterjea, M., y Shinde, R., Textbook of medical biochemistry, Octava ed., Jaypee Brothers Medical Publishers, (2012)

Chau, C.F., y Huang, Y.L., Characterization of passion fruit seed libres: a potential fibre source, https://doi.org/10.1016/j.foodchem.2003.05.009, Food Chemistry, 85(2), 189-194 (2004)

Chen, H.Y., y Yen, G.C., Antioxidant activity and free radical-scavenging capacity of extracts from guava (Psidium guajava L.) leaves, https://doi.org/10.1016/j.foodchem.2006.02.047, Food Chemistry, 101(2), 686-694 (2007)

Contreras-Calderón, J., Calderón-Jaimes, L., Guerra-Hernández, E., y García-Villanova, B., Antioxidant capacity, phenolic content and vitamin $\mathrm{C}$ in pulp, peel and seed from 24 exotic fruits from Colombia, https://doi.org/10.1016/j.foodres.2010.11.003, Food Research International, 44(7), 2047-2053 (2011)

Coultate, T., Manual de química y bioquímica de los alimentos, Acribia, Zaragoza, España, (2002)

Cuerda, C., Luengo, L.M., y otros cinco autores, Antioxidantes y diabetes mellitus: revisión de la evidencia, https://doi.org/10.3305/nh.2011.26.1.5115, Nutricion Hospitalaria, 26(1), 68-78 (2011) 
Deng, J., Liu, Q., Zhang, Q., y otros cuatro autores, Comparative study on composition, physicochemical and antioxidant characteristics of different varieties of kiwifruit seed oil in China, https://doi.org/10.1016/j.foodchem.2018.05.063, Food Chemistry, 264, 411-418 (2018)

European Union Commission, Regulation EEC 2568/91 on the characteristics of olive oil and olive-residue oil and on the relevant methods of analysis, Official Journal of European Communities, 34 (L 248), (1991)

Franco-Mora, O., Salomón-Castaño, J., Morales, A.A. y Castañeda-Vildózola, Á.R., Ácidos grasos y parámetros de calidad del aceite de semilla de uva silvestre (Vitis spp.), http://dx.doi.org/10.17268/sci.agropecu.2015.04.04, Scientia Agropecuaria, 6 (4), 271-278 (2015)

Ghafoor, K., Özcan, M.M., y otros tres autores, Changes in quality, bioactive compounds, fatty acids, tocopherols, and phenolic composition in oven-and microwave-roasted poppy seeds and oil, https://doi.org/10.1016/j.Iwt.2018.10.017, LWT, 99, 490-496 (2019)

Holub, B.J., Clinical nutrition: 4. Omega-3 fatty acids in cardiovascular care, Cmaj, 166(5), 608-615 (2002) Jones, P.J., Clinical nutrition: 7. Functional foods—more than just nutrition, Cmaj, 166(12), 1555-1563 (2002)

Lafont, J.J., Páez, M.S. y Lans, E., Composición fisicoquímica de la semilla y del aceite de la semilla del Canime (Copaifera officinalis L.), https://dx.doi.org/10.4067/S0718-07642011000300004, Información Tecnológica, 22(3), 19-26 (2011)

Lafont, J.J., Espitia, A.A., y Páez, M.S., Estudio fisicoquímico del aceite y análisis proximal de la torta de semillas oleaginosas nativas de Córdoba-Colombia, https://dx.doi.org/10.4067/S0718-07642019000400085, Información Tecnológica, 30(4), 85-92 (2019)

Mesa-Vanegas, A.M., Zapata-Uribe, S., y otros cuatro autores, Actividad antioxidante de extractos de diferente polaridad de Ageratum conyzoides L., Boletín Latinoamericano y del Caribe de Plantas Medicinales y Aromáticas, 14(1), 1-10 (2015)

Muñoz, A., Ramos, F., y otros cinco autores, Evaluación del contenido de fitoesteroles, compuestos fenólicos y métodos químicos para determinar la actividad antioxidante en semilla de sacha inchi (Plukenetia volubilis L.), Revista de la Sociedad Química del Perú, 76(3), 234-241 (2010)

Murga, R., Sanz, M.T., Beltrán, S. y Cabezas, J.L., Solubility of some phenolic compounds contained in grape seeds, in supercritical carbon dioxide, https://doi.org/10.1016/S0896-8446(02)00033-5, The Journal of Supercritical Fluids, 23(2), 113-121 (2002)

Nazzaro, F., Fratianni, F., y otros seis autores, Antibacterial activity of three extra virgin olive oils of the campania region, southern italy, related to their polyphenol content and composition,

https://doi.org/10.3390/microorganisms7090321, Microorganisms, 7(9), 321 (2019)

Neuza, J., Da Silva, C., y Aranha, C., Antioxidant activity of oils extracted from orange (Citrus sinensis) seeds, http://dx.doi.org/10.1590/0001-3765201620140562, An. Acad. Bras. Cienc., 88 (2), 951-958 (2016)

Pantoja-Chamorro, A.L., Hurtado-Benavides, A.M. y Martínez-Correa, H.A., Evaluación del rendimiento, composición y actividad antioxidante de aceite de semillas de mora (Rubus glaucus) extraído con CO2 supercrítico, https://dx.doi.org/10.4067/S0718-07642017000100005, Información Tecnológica, 28(1), 35-46 (2017)

Ramadan, M.F., y Moersel, J.T., Impact of enzymatic treatment on chemical composition, physicochemical properties and radical scavenging activity of goldenberry (Physalis peruviana L.) juice, https://doi.org/10.1002/jsfa.2728, Journal of the Science of Food and Agriculture, 87(3), 452-460 (2007)

Ramos-Escudero, F., González-Miret, M.L., Viñas-Ospino, A., y Escudero, M.R., Quality, stability, carotenoids and chromatic parameters of commercial sacha inchi oil originating from peruvian cultivars, https://doi.org/10.1007/s13197019-03960-x, Journal of Food Science and Technology, 56(11), 4901-4910 (2019)

Saini, R.K., y Keum, Y.S., Omega-3 and omega- 6 polyunsaturated fatty acids: Dietary sources, metabolism, and significance-A review, https://doi.org/10.1016/j.lfs.2018.04.049, Life Sciences, 203, 255-267 (2018)

Selani, M.M., Contreras, C.J., y otros cuatro autores, Wine industry residues extracts as natural antioxidants in raw and cooked chicken meat during frozen storage, https://doi.org/10.1016/j.meatsci.2011.01.017, Meat Science, 88(3), 397-403 (2011)

Seukep, A.J., Fan, M., y otros tres autores., Plukenetia huayllabambana fruits: analysis of bioactive compounds, antibacterial activity and relative action mechanisms, https://doi.org/10.3390/plants9091111, Plants, 9(9), 1111 (2020)

Stupp, T., Freitas, R.A., y otros cuatro autores, Characterization and potential uses of Copaifera langsdorfii seeds and seed oil, https://doi.org/10.1016/j.biortech.2007.04.051, Bioresource Technology, 99(7), 2659-2663 (2008)

Sümeye, P., y Baştürk, A., Volatile compounds, fatty acid composition and antioxidant activity of Centaurea albonitens and Centaurea balsamita seeds growing in Van, Turkey, https://doi.org/10.9734/ejnfs/2019/v11i430161, EJNFS, 11(4), 187-199 (2019) 